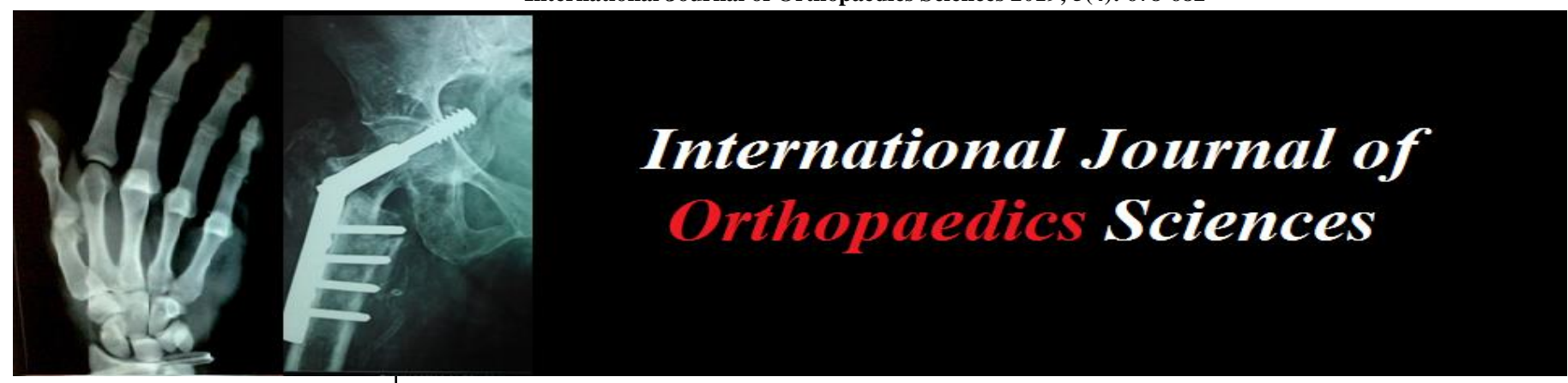

E-ISSN: 2395-1958

P-ISSN: 2706-6630

IJOS 2019; 5(4): 678-682

(C) 2019 IJOS

www.orthopaper.com

Received: 14-08-2019

Accepted: 18-09-2019

Gopu Govindasamy

Professor, Department of

Surgical Oncology, Government Royapettah Hospital, (Affiliated to Kilpauk Medical College), Royapettah, Chennai, Tamil Nadu, India
Corresponding Author: Gopu Govindasamy Professor, Department of Surgical Oncology, Government Royapettah Hospital, (Affiliated to Kilpauk Medical College), Royapettah, Chennai, Tamil Nadu, India

\section{Two stage revision limb salvage procedure for a malignant bone tumour following periprosthetic infection: A case report and review of literature}

\section{Gopu Govindasamy}

DOI: https://doi.org/10.22271/ortho.2019.v5.i41.1753

\section{Abstract}

Limb salvage surgery with endoprosthetic reconstruction is the standard of care in the management of extremity sarcomas of bone and soft tissues. Furthermore, adding neoadjuvant chemotherapy to the treatment protocol has increased the rates of limb salvage surgeries. Periprosthetic infection is an undesirable complication of the surgery the management of which is very challenging. Patients with Periprosthetic Joint Infection are treated in stages, firstly the prosthesis is removed with thorough debridement with or without placement of spacer followed by antibiotic treatment and later reimplantation of new prosthesis. Two stage procedure has been shown to have better results compared to single stage treatment of periprosthetic infection. Here we describe the case report of a patient who had osteochondroma of the right proximal tibia, treated with proximal tibial resection and custom mega prosthesis reconstruction and he had periprosthetic infection which treated in two stages and a review of literature of the treatment of periprosthetic joint infection.

Keywords: periprosthetic, reconstruction, infection, spacer

\section{Introduction}

Today, $90-95 \%$ of patients with extremity sarcomas are being treated with limb salvage surgery, most commonly by using endo- prosthetic implants. Periprosthetic infection remains one of the most challenging conditions to treat after endo- prosthetic reconstruction for patients and surgeons. The 'gold standard' treatment for this condition is two-stage revision arthroplasty, with removal of the prosthesis and spacer placement in the first stage, followed by antibiotic treatment, and then placement of a new prosthesis in the second stage with cure rates in the $80-100 \%$ range ${ }^{[2-11]}$.

The purpose of presenting this case is that as of now, to our knowledge currently there is no available case reports regarding two stage revision with custom made prosthesis in malignant bone tumour patients.

\section{Case description}

A 24 years old gentle man admitted with complaints of painful swelling in right proximal third leg for 3 months duration, gradual onset of swelling associated with pain. on examination, a bony hard swelling of size $15 * 10 \mathrm{cms}$ in right proximal tibia, more in posterior aspect without any distal neurovascular deficit and functional disability. MRI local part showed features in favour of osteochondroma in right proximal tibia with malignant transformation. Image guided biopsy was done from the bony tumour and reported as chondroid neoplasm. After getting assessment right proximal tibial resection and custom-made prosthesis reconstruction was done. Immediate postoperative periods were uneventful, drain removed on $8^{\text {th }}$ postoperative day, and sutures removed on 13 th postoperative day and patient rehabilitated with partial weight bearing started on 21st day and patient discharged. Patient was on regular follow up and rehabilitation. 4 months after primary surgery he developed a discharging sinus with pain in the operated site. He was admitted and initially treated with iv antibiotics according to pus culture reported as E. coli, after 10 days of conservative line of management patient was taken for wounds debridement and retention of prosthesis and discharged after a week. 
Then again after 6 weeks patient had persistent discharge through the sinus and patient readmitted and second surgery (prosthesis removal and antibiotic impregnated static spacer placement) done under cover of systemic antibiotics. After 10 weeks of second surgery revision custom made prosthesis implantation done after controlling the infective focus from the wound. Post-operative period was uneventful and rehabilitation exercises started. During follow up period he developed multiple discharging sinuses through the surgical scar site, treated initially conservatively, then High thigh above knee Amputation done in view of improving the Quality of life.

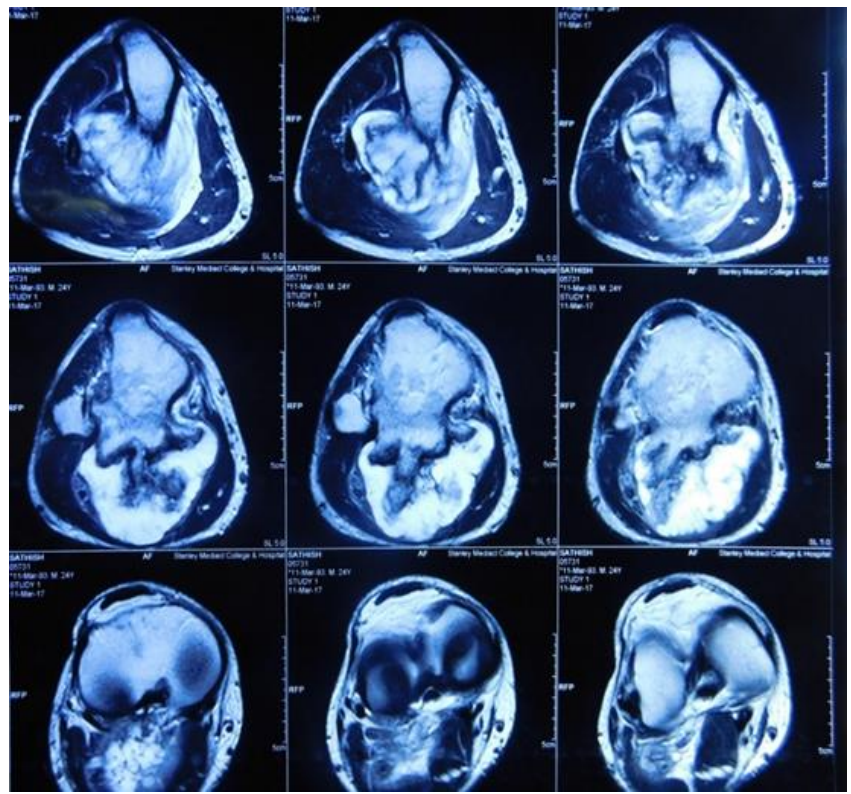

Fig 1: Chondrosarcoma of the left proximal tibia

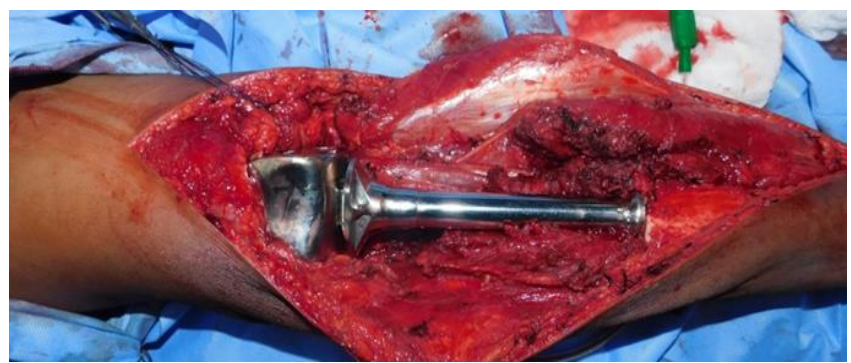

Fig 2: $1^{\text {st }}$ Surgery - Proximal tibial resection and custom mega prosthesis reconstruction

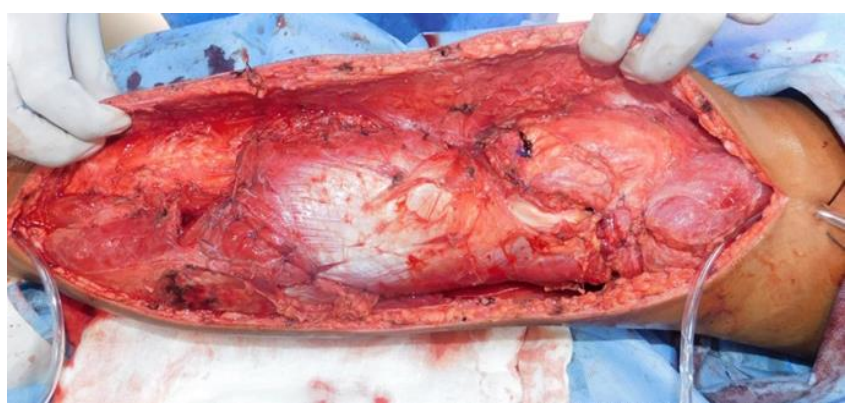

Fig 3: adequate soft tissue cover given after CMP placement

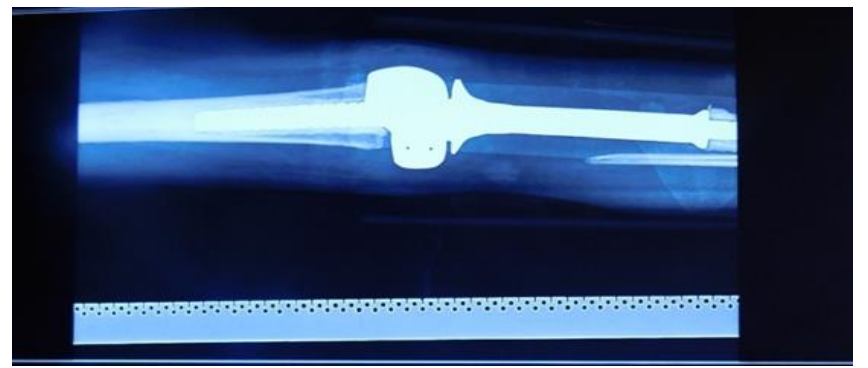

Fig 4: CMP in plain radiograph after $1^{\text {st }}$ Surgery

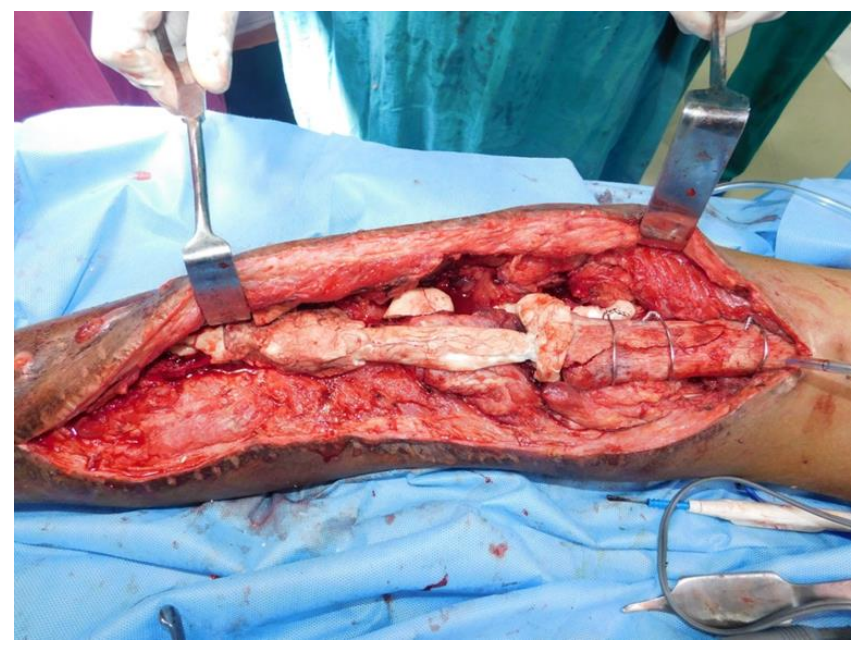

Fig 5: $2^{\text {nd }}$ Surgery - Exit of Prosthesis and placement of spacer

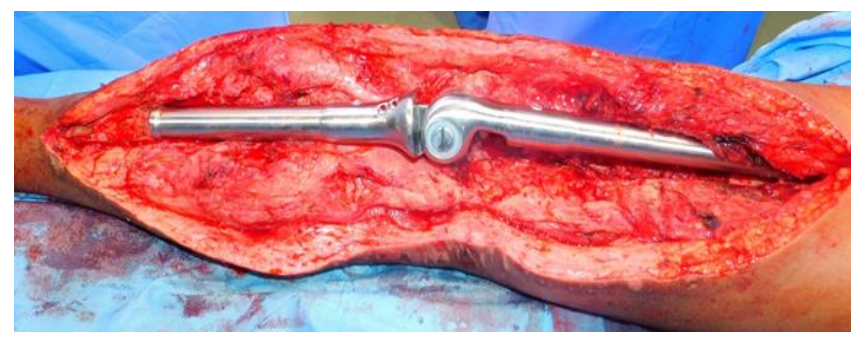

Fig 6: Spacer removal and placement of new CMP

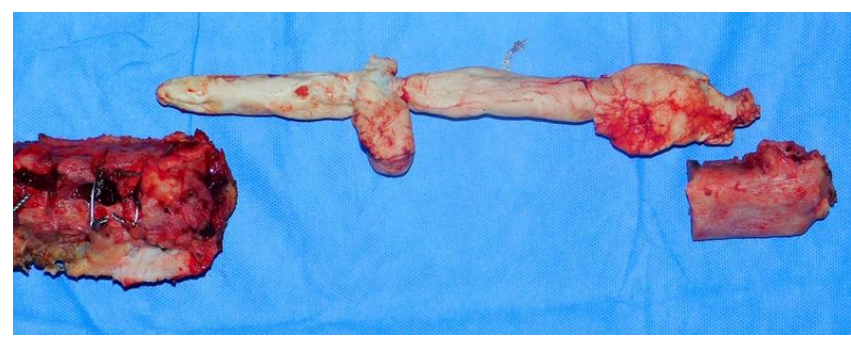

Fig 7: Spacer and the resected part of femur and tibia in the final surgey

\section{Discussion}

Management of patients with PJI treated with debridement and retention of the prosthesis, resection arthroplasty with or without subsequent staged reimplantation, 1-stage reimplantation, and amputation. Infection and septic failure are a devastating complication following primary surgical resection and endo-prosthetic reconstruction. 
Costs to society and the health care system can be as high as $\$ 566$ million per year, and is projected to exceed $\$ 1.62$ billion by 2020 [ 1 ford]. There is a $1-2 \%$ risk of infection following primary THA and the risk can be as high as $17 \%$ following revision THA ${ }^{[12-14]}$. Periprosthetic joint infections (PJI) is divided into either acute ( $<90$ day from index surgery or onset of symptoms) or chronic (>90 days) ${ }^{[15]}$. The definition of PJI has been divided into major and minor criteria, with the presence of either one major criteria or three minor criteria constituting $\mathrm{PJI}{ }^{[15]}$. Major criteria are the presence of a sinus tract communicating with the prosthesis or a positive culture from two or more separate tissue or fluid samples $[15,16]$. Minor criteria include elevated ESR and CRP; elevated synovial leukocyte count; elevated leukocyte neutrophil percentage (PMN \%); isolation of a microorganism in one culture of tissue or fluid; and > 5 neutrophils per high-power field in 5 high-power fields ${ }^{[15]}$. Two-stage revision is considered the gold standard of treatment. Insall et al. ${ }^{[16]}$ first described the technique in 1983. Garvin and Hanssen [17] conducted a review of 25 studies in 1995 showing an $82 \%$ success rate of two-stage revision compared to $58 \%$ with one-stage revision. Numerous studies have reported that twostage revision with use of antibiotic spacers can result in infection eradication rates as high as 95\% [18-22], although a recent study by Ford et al. ${ }^{[23]}$ reported only a $72 \%$ success rate in 66 patients.

In most circumstances, this involves resection of the prosthesis with or without placement of an antibiotic spacer, antibiotic treatment, following the patient's response to treatment and re-implantation of a new prosthesis. The firststage procedure involves thorough debridement of all lingering sources of infection within the joint cavity. This includes all components used in the index procedure, cement, and inflamed tissue within the joint. Antibiotic-impregnated cement is then used to create the spacer of choice to deliver local antibiotics and is used in conjunction with systemic antibiotics to target any isolated organisms. It is important to think about the type of antibiotic used in a spacer. Desirable characteristics include its availability in powder form, wide antibacterial spectrum, elution from bone cement in high concentrations, thermal stability, and low influence on the mechanical properties of the cement. Typically, vancomycin and aminoglycosides are used as they exhibit these properties. It is recommended that at $3.6 \mathrm{~g}$ of antibiotic be present per 40 $\mathrm{g}$ batch of cement ${ }^{[18,24]}$. The surgeon has many choices of spacer at their disposal including both static and mobile spacers options. Patients are then treated with systemic antibiotics targeted at the isolated organism. Many of surgeon's preference is to use a mobile spacer when the patient's bone stock and soft tissues are amenable. In terms of cement, the many of surgeons prefer to use PMMA that contains $1 \mathrm{~g}$ of Tobramycin (Simplex P, Stryker) and to that 3 $\mathrm{g}$ of Vancomycin are added and hand mixed. Classically, a 6week regimen of systemic antibiotics has been used ${ }^{[16]}$, and this is the Insall et al.'s preferential length of treatment; however, controversy exists in the literature. There is a paucity of convincing studies to guide an exact cut-off value for ESR, CRP, or synovial WBC count to guide timing of second-stage re-implantation. As long as there is a downtrend in values and there are no clinical signs of infection, the patients are then considered for re-implantation. During second-stage surgery, frozen-section analysis is obtained. If frozen section analysis is positive (based on the surgeon's preferred criteria), then the first-stage surgery is repeated and a new spacer placed, followed by a repeat course of systemic antibiotics in attempt to clear the infection. hsieh et al. ${ }^{\text {[25] }}$ reported Gram Negative (GN) PJI accounts for a substantial percentage of all PJI episodes and hence the choice of antimicrobial therapy for PJI should take into account the possibility of GN infection, especially in suspected patients who are older and who develop PJI early in the postoperative period. In comparison with PJI due to GP (gram positive) pathogens, GN PJI treated with 2- stage exchange and resection arthroplasty is associated with a comparatively favourable outcome. However, debridement and retention for GN PJI carries a higher rate of treatment failure and should be used sparingly among patients within the first few days after the onset of symptoms as seen in our case. Jmsen et al. ${ }^{[26]}$ reported that Mobile spacers are thought to ease patients' ambulation and prevent soft tissue contractures and muscle atrophy.

Despite the relatively high number of reports on the treatment of infected TKAs with exchange arthroplasty, there is a lack of prospective comparative trials. Most of the studies reviewed were retrospective case series and failed to describe materials, surgical technique, or both in enough detail- thus making it difficult to compare the studies to each other. The studies published so far (Hart and Jones 2006, Huang et al. 2006, Jämsen et al. 2006, Pietsch et al. 2006, Souillac et al. 2006, Trezies et al. 2006, Hsu et al. 2007, Mittal et al. 2007) do not appear to be any better in this respect. There was-and there still is - no question about the role of the two-stage approach being the gold standard in the management of infected knee replacements. Riesgo ${ }^{[27]}$ reported Local wound vancomycin powder has been shown in several large retrospective studies to reduce the rate of infection in spine surgery ${ }^{[27-32]}$. Additionally, these studies demonstrated the safety and efficacy of local vancomycin powder as prophylaxis against infection. The largest of these studies showed infection rates that decreased from $2.6 \%$ to $0.2 \%$ [27], while in a higher risk population of patients with a history of spinal trauma, a decrease in infection rate from $13 \%$ to $0 \%$ with the use of vancomycin powder was observed. In 2002 Taggart et al. ${ }^{[33]}$ reported the results of 33 arthroplasties (26 hips and 7 knees) which had performed a two stage revision procedure implemented by the use of vancomycin impregnated cement beads for infection caused by different organisms; after a mean follow-up of 67 months, 32 patient remained clinically and radiologically free from infection. The authors concluded that vancomycin played a major role in the management of infection after arthroplasties.

\section{Conclusion}

Although revision staged prosthesis reconstruction is commonly used in benign conditions, like in our case, it can also be used in malignant tumours. In our case, inspite of standardised treatment given, ultimately, the infection overwhelmed our effort. With this single case report we cannot conclude that amputation would be the ultimate solution for post-prosthetic complications, still there is an option for two stage revision limb salvage procedure. And further studies are warranted to support the usage of two-stage revision limb salvage procedures in malignant bone tumours.

\section{References}

1. Kurtz SM, Lau E, Watson H, Schmier JK, Parvizi J. Economic burden of periprosthetic joint infection in the United States. J Arthroplasty. 2012; 27(8):61-5.

2. Mortazavi SMJ, Vegari D, Ho A, Zmistowski B, Parvizi J. Two-stage Exchange Arthroplasty for Infected Total 
Knee Arthroplasty: Predictors of Failure. Clinical Orthopaedics and Related Research. 2011; 469(11):30493054. DOI: 10.1007/s11999-011-2030-8.

3. Sherrell JC, Fehring TK, Odum S et al. Fate of Twostage Reimplantation After Failed Irrigation and Débridement for Periprosthetic Knee Infection. Clinical Orthopaedics and Related Research. 2011; 469(1):18-25. DOI: 10.1007/s11999-010-1434-1.

4. Mittal Y, Fehring TK, Hanssen A, Marculescu C, Odum SM, Osmon D. Two-Stage Reimplantation for Periprosthetic Knee Infection Involving Resistant Organisms. J Bone Joint Surg Am. 2007; 89(6):1-5. DOI: 10.2106/JBJS

5. Lim SJ, Park JC, Moon YW, Park YS. Treatment of Periprosthetic Hip Infection Caused by Resistant Microorganisms Using 2-Stage Reimplantation Protocol. Journal of Arthroplasty. 2009; 24(8):1264-1269. DOI: 10.1016/j.arth.2009.05.012.

6. Hsieh PH, Shih CH, Chang YH, Lee MS, Shih HN, Yang WE. Two-Stage Revision Hip Arthroplasty for Infection: Comparison between the Interim Use of Antibiotic Loaded Cement Beads and a Spacer Prosthesis. J Bone Joint Surg Am. 2004, 1-9.

7. Volin SJ, Hinrichs SH, Garvin KL. Two-stage Reimplantation of Total Joint Infections. Clinical Orthopaedics and Related Research. 2004; 427:94-100. DOI: 10.1097/01.blo.0000143559.34143.3d.

8. Silvestre A1, Almeida F, Renovell P, Morante E, López R. Revision of Infected Total Knee Arthroplasty: TwoStage Reimplantation Using an Antibiotic-Impregnated Static Spacer. Clin Orthop Surg. 2013; 5(3):180-7.

9. Berbari EF, Marculescu C, Sia I et al. Culture-Negative Prosthetic Joint Infection. Clinical Infectious Diseases. 2007; 45(9):1113-1119. DOI: 10.1086/522184.

10. Kaminski A, Citak M, Schildhauer T, Fehmer T. Success Rates for Initial Eradication of Peri prosthetic Knee Infection Treated with a Two-stage Procedure. Ortop 217 Traumatol Rehabil. 2014; 16(1):11-16. DOI: 10.5604/15093492.1097485.

11. Chen AF, Heller S, Parvizi J. Prosthetic Joint Infections. Surg Clin North Am. 2014; 94(6):1265-1281. DOI: 10.1016/j.suc.2014.08.009.

12. Salvati E, Gonzalez Della Valle A, Masri B, Duncan C. The infected total hip arthroplasty. Instr Course Lect. 2003; 52:223-45.

13. Spangehl M, Masri B, O'Connell J, Duncan C. Prospective analysis of preoperative and intraoperative. Investigations for the diagnosis of infection at the sites of two hundred and. two revision total hip arthroplasties. J Bone Joint Surg Am. 1999; 81(5):672-83.

14. Springer B, Cahue S, Etkin C, Lewallen D, McGrory B. Infection burden in total hip and knee arthroplasties: an international registry based perspective. Arthroplasty Today. 2017; 3(2):137-40.

15. Parvizi J, Gehrke T. Definition of Periprosthetic joint infection. J Arthroplast. This article presents the Musculoskeletal Infection Society definition of both acute and chronic PJI. The article outlines the major and minor criteriafor PJI as well as cutoff values for synovial WBC count, PMN\%, ESR, CRP, Leukocyte esterase and histological analysis of tissue. 2014; 29:1331.

16. Insall $\mathrm{J}$, Thompson $\mathrm{F}$, Brause $\mathrm{B}$. Two-stage reimplantation for the salvage of infected total knee arthroplasty. J Bone Joint Surg Am. 1983; 65:1087-98.

17. Garvin K, Hanssen A. Infection after total hip arthroplasty: past, present, and future. J Bone Joint Surg Am. 1995; 77:1576-88.

18. Lu J, Han J, Zhang C, Yang Y, Yao Z. Infection after total knee arthroplasty and its gold standard surgical treatment: spacers used in two-stage revision arthroplasty. Intractable Rare Dis Res. 2017; 6(4):256-61.

19. Kalore N, Gioe T, Sing J. Diagnosis and management of infected total knee arthroplasty. Open Orthop J. 2011; 5:86-91.

20. Martinez-Pastor J, Macule-Beneyto F, Suso-Vergara S. Acute infection in total knee arthroplasty: diagnosis and treatment. Open Orthop J. 2013; 7:197-204.

21. Chun K, Kim K, Chun C. Infection following total knee arthropalsty. Knee Surg Relat Res. 2013; 25:93-9.

22. De Carvalho L, Tempon E, Badet R. Infection after total knee replacement: diagnosis and treatment. Rev Bras Orthop. 2013; 24:389-96.

23. Ford A, Holzmeister A, Rees H, Pelich B. Characterization of outcomes of 2-stage exchange arthroplasty in the treatment of prosthetic joint infections. J Arthroplasty, 2018, https://doi.org/10.1016/j.arth.002018.000002.000043.

This is a recent review of 80patients undergoing resection arthroplasty with placement of an antibiotic spacer for PJI at a single institution. They report lower rates of success than previously reported in the literature, including only 66 of the patients undergoing second-stage reimplantation, and $28 \%$ of these patients having persistent infection. Additionally, $30 \%$ of patients had a serious complication during their treatment course

24. Jacobs C, Christian C, Berend M. Static and mobile antibiotic impregnated cement spacers for the management of prosthetic joint infection. J Am Acad Orthop Surg. 2009; 17:356-8.

25. Gram-Negative Prosthetic Joint Infections: Risk Factors and Outcome of Treatment Pang-Hsin Hsieh, 1, 2 Mel S. Lee, 1, 2 Kuo-Yao Hsu, 1, 2 Yu-Han Chang, 1, 2 HsinNung Shih, 1, 2 and Steve W. Ueng1, 21 Department of Orthopedic Surgery, Chang Gung Memorial Hospital, and 2 College of Medicine, Chang Gung University, Taoyuan, Taiwan.

26. Outcome of prosthesis exchange for infected knee arthroplasty: the effect of treatment approach A systematic review of the literature Esa Jämsen1, 2, Ioannis Stogiannidis1, 2, Antti Malmivaara3, Jorma Pajamäki2, Timo Puolakka2, and Yrjö T Konttinen2, 4.

27. Sweet FA, Roh M, Sliva C. Intrawound application of vancomycin for prophylaxis in instrumented thoracolumbar fusions: efficacy, drug levels, and patient outcomes. Spine (Phila Pa 1976). 2011; 36(24):2084-8.

28. Molinari RW, Khera OA, Molinari WJ. 3rd, Prophylactic intraoperative powdered vancomycin and postoperative deep spinal wound infection: 1, 512 Consecutive surgical cases over a 6-year period. Eur Spine J. 2012; 21(Suppl 4):S476-82.

29. O'Neill KR et al., Reduced surgical site infections in Patients undergoing posterior spinal stabilization of traumatic injuries using vancomycin powder. Spine J. 2011; 11(7):641-6.

30. Caroom $\mathrm{C}$ et al., Intrawound vancomycin powder reduces surgical site infections in posterior cervical fusion. Spine (Phila Pa 1976). 2013; 38(14):1183-7.

31. Strom RG et al., Decreased risk of wound infection after posterior cervical fusion with routine local application of vancomycin powder. Spine (Phila Pa1976), 2013. 
32. Strom RG et al., Lumbar laminectomy and fusion with routine local application of vancomycin powder: decreased infection rate in instrumented and noninstrumented cases. Clin Neurol Neurosurg. 2013; 115(9):1766-9.

33. Taggart T, Kerry RM, Norman P, Stockley I. The use of vancomycin-impregnated cement beads in the management of infection of prosthetic joints. $\mathrm{J}$ Bone $\mathrm{Jt}$ Surg Br. 2002; 84-B (1):70-72. 